Mens

revue d'histoire intellectuelle de l'Amérique française

\title{
À propos d'une lecture du citoyen Cook
}

\section{Yvan Lamonde}

Volume 11, numéro 1, automne 2010

URI : https://id.erudit.org/iderudit/1023339ar

DOI : https://doi.org/10.7202/1023339ar

Aller au sommaire du numéro

Éditeur(s)

Centre de recherche en civilisation canadienne-française

ISSN

1492-8647 (imprimé)

1927-9299 (numérique)

Découvrir la revue

Citer ce document

Lamonde, Y. (2010). À propos d'une lecture du citoyen Cook. Mens, 11(1), 95-99.

https://doi.org/10.7202/1023339ar d'utilisation que vous pouvez consulter en ligne.

https://apropos.erudit.org/fr/usagers/politique-dutilisation/ 


\title{
Répliques
}

\section{À propos d'une lecture du citoyen Cook}

\author{
Yvan Lamonde \\ Département de langue et littérature françaises \\ Université McGill
}

Je choisis de m'entretenir ici avec Ramsay Cook parce qu'il lit et respecte les historiens québécois, ce qui n'est pas le schéma dominant de l'historiographie canadienne. On peut ne pas être d'accord avec ses analyses ou ses interprétations, c'est là le pain quotidien de la recherche. Mais pour qui, comme moi, n'est pas un militant ni un historien "marqué " par la récurrence des interventions publiques ou partisanes, la connaissance et l'intelligence historiques de R. Cook ne peuvent que susciter une relance de la réflexion.

\section{À propos de " présentisme "}

J'aimerais d'abord tenter de dissiper une confusion à propos de l'idée courante de "présentisme ». C'est une chose de reconnaître avec et après Lucien Febvre que l'historien ne peut pas ne pas lire l'outillage mental des acteurs du passé en prétendant gommer l'outillage mental qui est le sien comme lecteur du passé. Cela peut aussi s'appeler la sensibilité propre de l'historien, l'antenne de perception irrécusable qui est la sienne. Le présentisme est une lecture habituellement peu critique où l'on passe l'aimant du présent sur la ferraille du passé, où l'on plaque sur le passé un questionnement, un 
vocabulaire contemporains, où l'on cherche dans un passé plus ou moins lointain des " preuves » d'une thèse militante contemporaine.

Ma démarche n'a pas été de cet ordre pour autant que je puisse l'affirmer, conscient que je suis d'être, ici, juge et partie. J'irai aussi loin que d'essayer de nommer les sensibilités qui devinrent les miennes, sensibilités qui ne m'étaient pas connues ou transparentes au départ, mais qui le sont devenues en fin de compte. Je pense avoir regardé le passé, sans m'en rendre compte clairement durant le processus, avec un certain bagage mental de Cité libre - critique du cléricalisme, place de la démocratie dans la société canadienne-française - et de l'historiographie sociale qu' incarnait Jean Hamelin, où une certaine histoire sociale - celle des structures sociales et des groupes sociaux - devait traverser d'une façon ou d'une autre une problématique et une analyse historiques. Cette lecture sociale n'était pas pour autant celle de Parti pris. Non que je la récuse - sa marque forte sur ses analyses a fait voir des choses qui n'avaient pas été suffisamment ou clairement définies avant -, mais c'est précisément son " présentisme militant » qui ne m'attirait pas comme historien.

\section{Ma navigation}

C'est après avoir terminé les trois premiers tomes de mon Histoire sociale des idées au Québec (Fides) que j'ai pu voir quelle lecture différente du passé ce travail avait occasionnée. De la même façon, la formule que cite R. Cook $\left(\mathrm{Q}=-\mathrm{F}+\mathrm{GB}+\mathrm{USA}^{2}-\mathrm{R}\right)$ ne fut pas un point de départ, mais un point d'arrivée. Cette lecture différente du passé me faisait voir certains aspects du présent et je ne prétendais pas, dans Historien et citoyen (Fides, 2008), dire ce que je pensais comme historien de toute l'histoire contemporaine du Québec, de 1965 à 2010. J'en dirai plus long dans le bouquin qui vient de paraître, L'heure de vérité: la laicité québécoise à l'épreuve de l'histoire (Del Busso Éditeur, 2010), mais ce ne sera pas pour autant, encore une fois, une analyse globale du contemporain. 
Je ne dis rien sur les allophones dans Historien et citoyen - bien que je fasse place aux Juifs et aux Italiens dans les tomes 2 et 3 de l'histoire sociale des idées - parce que, dans ce que j'ai analysé, leur présence émergeait sans avoir encore de poids relatif significatif. Je sais bien qu'une histoire de l'immigration et des groupes immigrés manque au Québec - sur le modèle de l'ouvrage dirigé par Robert Magocsi, Encyclopedia of Canada's Peoples (University of Toronto Press, 1999) - ; j'en lance l'idée comme une bouteille à la mer qu'on pourra s'approprier.

Je pense avoir essentiellement dit que, contrairement à ce qu'avait répété Pierre Elliott Trudeau, il y avait une tradition démocratique au Québec, que mes contemporains devaient en connaître l'existence, même limitée, et qu'ils pouvaient y puiser pour penser les défis d'une majorité francophone au Québec confrontée à la diversité de quelque nature qu'elle soit.

\section{La « normalité "}

Cette idée est issue de la critique faite par Ronald Rudin d'une certaine historiographie qui ferait de la société canadienne-française d'avant 1960 une société comparable à d'autres sociétés occidentales " modernes ", "normales ». R. Cook m'associe à Gérard Bouchard au sens où la préoccupation de "décolonisation " serait suspecte parce que participant d'un souci que le Québec devienne " normal ", décolonisé. En quoi et pourquoi cette préoccupation fréquente d'historiens québécois serait-elle irrecevable, illégitime? Je ne présente pas, par exemple, dans le tome III de l'Histoire sociale des idées au Québec (à paraître), l'indépendantisme des années 1930 comme une voie souhaitable, pas plus que je ne le ferai, dans le tome IV, en ce qui concerne la démarche indépendantiste et souverainiste des années 1950 et 1960. Mais il me faut voir, comme historien, que ce problème est posé PAR DES CONTEMPORAINS. Que l'on soit pour ou contre l'idée de décolonisation ou que cela ne plaise pas pour des raisons politiques ou idéologiques ne m’importent pas. Cette question 
historique a été posée, il faut en rendre compte sans que, du coup, l'historien qui le fait soit annexé à une cause. En fait, ce que j'aurai établi avec les quatre tomes de l'Histoire sociale des idées au Québec concerne la continuité d'une tradition d'émancipation politique, avec ses moments forts et ses creux, avec ses associations à la droite comme à la gauche. J'aurai aussi établi la continuité d'une tradition démocratique, la continuité d'une tradition laïque, elles aussi avec leurs moments faibles et leurs moments forts. Ce sera l'effet d'une SYNTHĖSE des courants et des débats d'idées que de faire voir la CONSTANCE de certaines préoccupations au fil des tentatives coloniales de diviser pour régner.

Je me demande si R. Cook fait place au colonialisme dans son analyse historique, à l'idée coloniale qui n'a qu'une intention véritable : coloniser, faire devenir un homme donné colon d'un autre homme, d'une autre puissance. Je reconnais qu'en comparaison avec d'autres stratégies colonialistes, le colonialisme britannique fut plus subtil. Mais il fit son travail. Qu'on veuille le nier, le minimiser, l'évacuer est tout aussi inacceptable que de vouloir le magnifier, l'instrumentaliser politiquement.

Alors pourquoi refuserait-on qu'il soit question de " décolonisation " au Québec à la fin des années 1950 et dans les années 1960 ? Parce que des historiens voudraient que le Québec soit normal ? Je n'ai pas besoin de cette raison pour analyser l'après-guerre ; cette décolonisation fait partie de la donne.

\section{La mémoire historique, " un donné contestable " ?}

Pour R. Cook, l'identité à laquelle je fais référence « repose sur une culture nourrie par la mémoire historique, un donné contestable qui n'est ni remis en question ni démontré1 ". Que comprendre ? Que la mémoire historique est un bien jetable, non durable? Qu'il ne s'agit toujours que de "tradition inventée ", de nations

1 Ramsay Cook, "L'éducation du citoyen Lamonde ", Mens : revue d'histoire intellectuelle et culturelle, vol. X, $\mathrm{n}^{\circ} 2$ (printemps 2010), p. 95. 
" imaginaires ", de " fragments " postmodernes, de navire sans ancre, de navire à la dérive ? Pour bien comprendre le propos de R. Cook, je souhaiterais qu'il m'éclaire éventuellement en répondant à la question suivante : y a-t-il une mémoire historique dans le multiculturalisme ou son absence est-elle la condition d'existence de celui-ci ?

\section{Réponse de Ramsay Cook à Yvan Lamonde}

Je tenterai de répondre brièvement, peut-être trop brièvement même, aux importantes questions soulevées par Yvan Lamonde dans sa réponse à ma recension de son ouvrage Historien et citoyen.

$\mathrm{Par}$ " présentisme », je fais référence à la téléologie implicite dans toute histoire nationale. Présupposer que les communautés possèdent des identités collectives qui évoluent naturellement vers le cadre national - que la finalité de cette évolution est donc connue d'avance - détermine les questions qui sont posées au passé et les jugements qui sont portés à son égard. Une grande partie des historiographies canadienne, canadienne-française et québécoise illustre, je crois, cette approche téléologique. Trop souvent les historiens "imagine the past and remember the future", pour reprendre l'expression de l'historien britannique Sir Lewis Namier. À tout le moins, l'histoire racontée aux Canadiens et aux Québécois a longtemps laissé dans l'ombre de grands pans du passé, si ce n'est la plus grande part, comme nous l'ont révélé les récentes générations d'historiens.

Parallèlement, il ne fait aucun doute que le concept de " colonialisme " permet de comprendre à la fois l'histoire de la NouvelleFrance et, d'une manière plus complexe et changeante, celle du Canada (aussi bien francophone qu'anglophone) de 1763 aux années 1920 - peut-être même à 1931. Après cette date, le statut autonome formellement concédé au Canada a su satisfaire aussi bien les Canadiens français que les Canadiens anglais. Ce qui est vrai pour 